Research, Society and Development, v. 9, n. 12, e37191211033, 2020

(CC BY 4.0) | ISSN 2525-3409 | DOI: http://dx.doi.org/10.33448/rsd-v9i12.11033

\title{
A dor crônica na hipertensão arterial em adultos: um estudo bibliométrico
}

Chronic pain in hypertension in adults: a bibliometric study

Dolor crónico en la hipertensión en adultos: un estudio bibliométrico

Recebido: 09/12/2020 | Revisado: 16/12/2020 | Aceito: 21/12/2020 | Publicado: 27/12/2020

Valeriana Cantanhede Rodrigues

ORCID: https://orcid.org/0000-0002-4921-2675

Universidade Federal Fluminense, Brasil

E-mail: valerianarodrigues@id.uff.br

Dayse Mary da Silva Correia

ORCID: https://orcid.org/0000-0002-6678-1378

Universidade Federal Fluminense, Brasil

E-mail: daysecorreia@id.uff.br

\section{Resumo}

Introdução: há uma relação de doenças crônicas não transmissíveis (DCNT) com a dor crônica. E como dentre essas doenças, há a estimativa que a hipertensão arterial acometa 36 milhões de brasileiros, infere-se a importância do estudo. Logo, o objetivo foi analisar os indicadores bibliométricos de dissertações e teses sobre dor cônica na hipertensão em território brasileiro. Metodologia: trata-se de um estudo bibliométrico realizado no Banco de Teses e Dissertações da Coordenação de Aperfeiçoamento de Pessoal de Nível Superior (CAPES) e na Biblioteca Digital Brasileira de Teses e Dissertações (BDTD), entre os anos de 2010 a 2020 no Brasil. A coleta de dados deu-se de outubro a novembro de 2020, utilizandose as palavras-chave "dor crônica" e "hipertensão", com elaboração posterior de um mapa conceitual a partir dos descritores das pesquisas. Resultados: dos 982 estudos identificados, somente 20 atenderam aos critérios de inclusão, dos quais 4 teses e 16 dissertações, com utilização da abordagem quantitativa. Considerações Finais: foi observado a relação da dor crônica e hipertensos, no entanto, porém evidenciando um pequeno número de pesquisas. Desse modo, faz-se necessário novas investigações.

Palavras-chave: Hipertensão; Dor crônica; Indicadores bibliométricos. 


\section{Abstract}

Introduction: there is a relationship between chronic non-communicable diseases (CNCD) and chronic pain. And as among these diseases, there is an estimate that hypertension affects 36 million Brazilians, the importance of the study is inferred. Therefore, the objective was to analyze the bibliometric indicators of dissertations and theses on conical pain in hypertension in Brazilian territory. Methodology: this is a bibliometric study carried out at the Bank of Theses and Dissertations of the Coordination for the Improvement of Higher Education Personnel (CAPES) and at the Brazilian Digital Library of Theses and Dissertations (BDTD), between the years 2010 to 2020 in Brazil. Data collection took place from October to November 2020, using the keywords "chronic pain" and "hypertension", with subsequent elaboration of a conceptual map from the research descriptors. Results: of the 982 studies identified, only 20 met the inclusion criteria, including 4 theses and 16 dissertations, using the quantitative approach. Final Considerations: the relationship between chronic pain and hypertension was observed, however, showing a small number of studies. Thus, further investigations are necessary.

Keywords: Hypertension; Chronic pain; Bibliometric indicators.

\section{Resumen}

Introducción: existe una relación entre las enfermedades crónicas no transmisibles (ECNT) y el dolor crónico. Y como entre estas enfermedades se estima que la hipertensión afecta a 36 millones de brasileños, se infiere la importancia del estudio. Por tanto, el objetivo fue analizar los indicadores bibliométricos de disertaciones y tesis sobre el dolor cónico en la hipertensión en territorio brasileño. Metodología: se trata de un estudio bibliométrico realizado en el Banco de Tesis y Disertaciones de la Coordinación para el Perfeccionamiento del Personal de Educación Superior (CAPES) y en la Biblioteca Digital Brasileña de Tesis y Disertaciones (BDTD), entre los años 2010 a 2020 en Brasil. La recolección de datos se llevó a cabo de octubre a noviembre de 2020, utilizando las palabras clave "dolor crónico" e "hipertensión", con posterior elaboración de un mapa conceptual a partir de los descriptores de la investigación. Resultados: de los 982 estudios identificados, solo 20 cumplieron con los criterios de inclusión, incluyendo 4 tesis y 16 disertaciones, utilizando el enfoque cuantitativo. Consideraciones Finales: se observó la relación entre dolor crónico e hipertensión, sin embargo, mostrando un número reducido de estudios. Por tanto, son necesarias más investigaciones. 
Palabras clave: Hipertensión; Dolor crónico; Indicadores bibliométricos.

\section{Introdução}

Dentre as Doenças Crônicas Não-Transmissíveis (DCNT), dados epidemiológicos apontam que hipertensão arterial (HA) está presente em 36 milhões de adultos no Brasil, representando $32,5 \%$ da população dessa faixa etária, e tendo entre idosos elevada prevalências, ou seja, em cerca de $60 \%$ da população acima dos 60 anos. Além disso, foi uma doença crônica responsável por $50 \%$ das mortes por doenças cardiovasculares no país (Brandão \& Nogueira, 2018). De acordo com a Sociedade Brasileira do Estado do Rio de Janeiro (SOCERJ), a hipertensão, pode ser conceituada como a elevação sustentada dos níveis pressóricos em valores iguais ou acima de 140x90 mmHg (Brandão \& Nogueira, 2018).

Cabe salientar, que há uma relação entre DCNT e dor crônica, pois em 2018 pelo menos $37 \%$ da população brasileira ou 60 milhões de pessoas relataram sentir dor (Albuquerque, 2018).

A dor é descrita como de difícil compreensão e multifatorial, definida pela International Association for the Study of Pain (IASP) como uma "experiência sensorial e emocional desagradável podendo estar associada a uma lesão real ou descrita em tais termos". Quando aguda, serve de alerta para ocorrências de lesões no corpo, já a dor crônica não possui características e tem-se que o melhor modo de diferenciação entre dor crônica e aguda são três meses de persistência do agravo (Witte \& Stein, 2010).

E é experienciada de forma subjetiva, e tem interferência de acordo com a percepção que cada indivíduo tem de sua vida. E ainda, requer ações adequadas visando o bem-estar e controle, a redução das queixas dolorosas para melhorar a capacidade funcional, a qualidade de vida e desenvolver estratégias preventivas que ajudem a melhorar a saúde desses indivíduos (Hlubocky et al., 2016).

Logo torna-se um parâmetro para a avaliação da qualidade de vida, pois muitas vezes estimula situações incomodas e limitantes (Freitas et al., 2012). Desse modo, a intensidade da dor acaba sendo fundamental para explorar a sua influência no modo de vida do ser humano.

Portanto, sendo prevalente no Brasil, a dor crônica, e ainda sob escassez do tema associado à hipertensão, fez-se necessário um estudo bibliométrico acerca da produção científica em cursos de pós-graduação stricto sensu.

Visto que, os estudos bibliométricos possibilitam o direcionamento de novas investigações sobre o tema estudado e auxiliam no alcance de indicadores da produção 
científica, identificando o tempo, a origem dos trabalhos, os temas e as metodologias empregadas (Dias et al., 2020)

Logo, este estudo tem por objetivo o de analisar os indicadores bibliométricos de dissertações e teses sobre dor cônica na hipertensão em território brasileiro.

\section{Metodologia}

Trata-se de um estudo bibliométrico, para o qual a análise bibliométrica em pesquisas científicas destinam-se na investigação do comportamento da evidência científica como parte dos processos de comunicação (Marcelo et al, 2013)

A pesquisa foi realizada na Biblioteca Digital Brasileira de Teses e Dissertações e no Catálogo de Teses e Dissertações Capes, com a utilização dos termos "dor crônica" and "hipertensão arterial" para a seleção da amostra. Em seguida, foram definidas como critérios de inclusão, as publicações nas modalidades de dissertações e teses, divulgadas no idioma português, entre os anos de 2010 e 2020 e caracterizados como critério de exclusão, os trabalhos que abordaram como tema, gestantes e crianças.

Por tratar-se de uma busca por estudos bibliométricos, é importante um recorte temporal que contemple um número maior de publicações de teses e dissertações para dispormos de uma amostra ampla, mas atual, tendo em vista um significativo quantitativo de estudos.

A coleta de dados foi realizada em um segundo momento, com o emprego de um instrumento elaborado pelas autoras, a fim de organizar a pesquisa, demonstrar a atividade científica produzida e gerar indicadores bibliométricos. $\mathrm{O}$ instrumento descreve as seguintes variáveis: título, modalidade de publicação, formação do pesquisador, nome da instituição, ano de defesa, região em que o estudo foi produzido, desenho metodológico, palavras-chaves e artigos desenvolvidos a partir das dissertações e teses. A coleta de dados aconteceu nos meses de setembro e outubro de 2020. Os dados foram obtidos por meio da leitura de títulos e resumos e quando estes não eram suficientes, os trabalhos eram lidos em sua totalidade.

Para a seleção dos estudos, seguiram-se as recomendações do checklist do Statement for Reporting Systematic Review and Meta-Analyses of Studies - PRISMA (Galvão et al., 2015), conforme a Figura 1. 
Research, Society and Development, v. 9, n. 12, e37191211033, 2020

(CC BY 4.0) | ISSN 2525-3409 | DOI: http://dx.doi.org/10.33448/rsd-v9i12.11033

Figura 1: Fluxograma do processo de seleção dos estudos, adaptado do PRISMA.

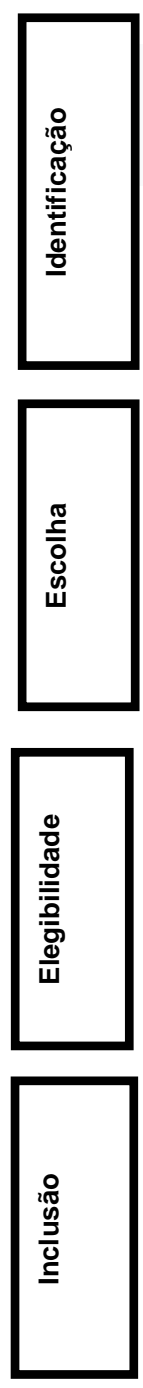

Dissertações e teses identificadas por meio de buscas em bases de dados $(n=982)$

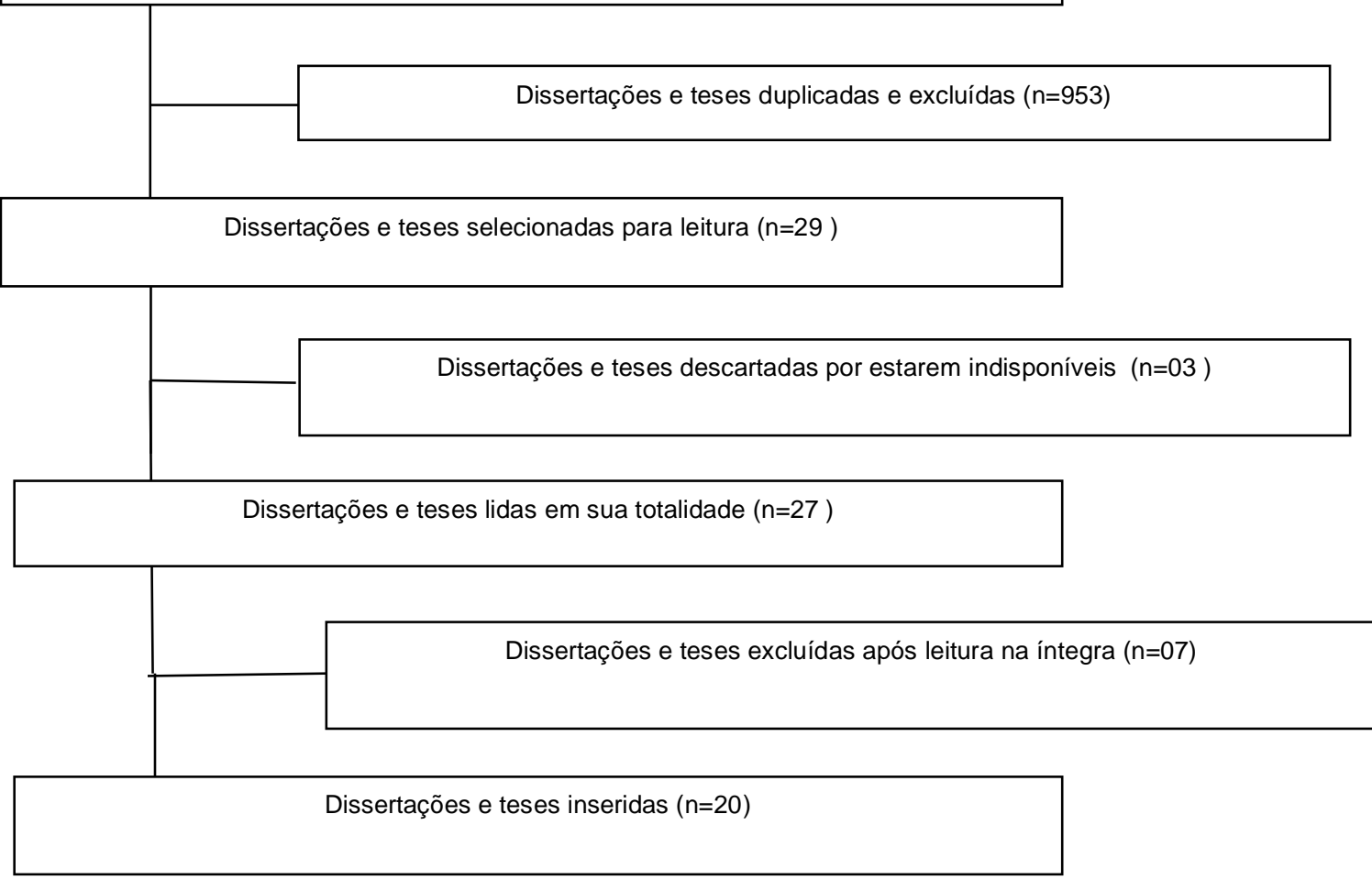

Fonte: Autores.

Os dados coletados a partir das buscas e leituras de teses e dissertações selecionadas para o estudo foram analisados com base no instrumento proposto para coleta de dados. Posteriormente, foram apresentados por meio de representações gráficas e analisados quantitativamente por meio de frequência absoluta e percentual por ferramenta do programa Microsoft Office Excel ® 2010.

Com a finalidade de organização das palavras-chaves, foi utilizado o mapa conceitual, ferramenta empregada para uma reprodução gráfica de conceitos interligados através de proposições e descritores. Nas pesquisas no campo da enfermagem, os mapas conceituais são utilizados com a função de estimular o pensamento crítico, contribuir para a solução de problemas, sintetizar e simplificar conceitos, com o objetivo de avaliar ações para um plano de cuidados efetivo (Alves et al., 2019). 
Research, Society and Development, v. 9, n. 12, e37191211033, 2020

(CC BY 4.0) | ISSN 2525-3409 | DOI: http://dx.doi.org/10.33448/rsd-v9i12.11033

No presente estudo, o mapa conceitual serviu para a associação da temática conceitual entre as palavras-chaves mencionadas nas dissertações e teses selecionadas e no contexto do estudo. As palavras-chave foram agrupadas a partir das principais - Dor Crônica e Hipertensão Arterial - por serem tema do estudo, e organizadas ordenadamente em classes temáticas.

\section{Resultados}

Quadro 1: Demonstração dos estudos acerca da temática dor crônica em hipertensos, com relação à região, instituição de ensino superior, programas de pós-graduação, dissertações e teses ( $\mathrm{n}=20)$. Rio de Janeiro, 2020.

\begin{tabular}{|c|c|c|c|c|}
\hline gião & Instituição / Programa de Pós-graduação & Dissertação & Tese & $\begin{array}{l}\text { Total } \\
\mathrm{n}(\%)\end{array}$ \\
\hline \multirow[b]{3}{*}{ Sul } & $\begin{array}{l}\text { Universidade Federal do Rio Grande do Sul } \\
\text { ( Ciências da Saúde: Cardiologia e Ciências Cardiovasculares) }\end{array}$ & 1 & 1 & $2(10)$ \\
\hline & $\begin{array}{l}\text { Universidade Federal de Santa Maria } \\
\text { (Mestrado em Educação Física) }\end{array}$ & 1 & 0 & $1(5)$ \\
\hline & $\begin{array}{c}\text { Universidade Estadual de Londrina } \\
\text { Mestrado de Enfermagem } \\
\text { Doutorado em Saúde Coletiva } \\
\text { Centro de Ciências da Saúde Programa de Pós-Graduação em Enfermagem }\end{array}$ & $\begin{array}{l}1 \\
0 \\
1\end{array}$ & $\begin{array}{l}0 \\
1 \\
0\end{array}$ & $\begin{array}{l}1(5) \\
1(5) \\
1(5)\end{array}$ \\
\hline \multirow[t]{3}{*}{ Sudeste } & $\begin{array}{c}\text { Universidade São Paulo } \\
\text { ( Tecnologias e Atenção à Saúde - Mestrado Profissional) } \\
\text { Mestrado em Enfermagem na Saúde do Adulto } \\
\text { Mestrado em Enfermagem Fundamental } \\
\text { Mestrado em Saúde Coletiva }\end{array}$ & $\begin{array}{l}1 \\
1 \\
1 \\
1\end{array}$ & $\begin{array}{l}0 \\
0 \\
0 \\
0\end{array}$ & $\begin{array}{l}1(5) \\
1(5) \\
1(5) \\
1(5)\end{array}$ \\
\hline & $\begin{array}{l}\text { Universidade do Estado do Rio de Janeiro } \\
\text { Mestrado em Saúde Coletiva }\end{array}$ & 1 & 0 & $1(5)$ \\
\hline & $\begin{array}{l}\text { Universidade Federal de Minas Gerais } \\
\text { (Doutorado em Medicações e Assistências Farmacêuticas) }\end{array}$ & 0 & 1 & $1(5)$ \\
\hline \multirow{3}{*}{ Centro-Oeste } & $\begin{array}{c}\text { Universidade de Brasília } \\
\text { Mestrado em Medicina } \\
\text { Doutorado em Ciências da Saúde - Saúde Coletiva } \\
\text { Mestrado - Programa de Pós-Graduação em Ciências e Tecnologias em Saúde }\end{array}$ & $\begin{array}{l}1 \\
0 \\
1\end{array}$ & $\begin{array}{l}0 \\
1 \\
0\end{array}$ & $\begin{array}{l}1(5) \\
1(5) \\
1(5)\end{array}$ \\
\hline & $\begin{array}{c}\text { Universidade Católica da Brasília } \\
\text { (Mestrado - Programa Stricto Sensu em Gerontologia - Escola de Saúde e } \\
\text { Medicina }\end{array}$ & 1 & 0 & $1(5)$ \\
\hline & $\begin{array}{c}\text { Universidade Federal de Goiás } \\
\text { Mestrado Profissional em Gestão Organizacional } \\
\text { Mestrado - Programa de Pós-graduação em Enfermagem }\end{array}$ & $\begin{array}{l}1 \\
1\end{array}$ & $\begin{array}{l}0 \\
0\end{array}$ & $\begin{array}{l}1(5) \\
1(5)\end{array}$ \\
\hline & Universidade Estadual do Ceará & & & \\
\hline
\end{tabular}


(CC BY 4.0) | ISSN 2525-3409 | DOI: http://dx.doi.org/10.33448/rsd-v9i12.11033

\begin{tabular}{|c|r|c|c|}
\multirow{2}{*}{ Nordeste } & Mestrado em Cuidados Clínicos em Enfermagem e Saúde & 1 & 0 \\
\cline { 2 - 5 } & Mestrado em Neuropsiquiatria e Ciências do Comportamento & $1(5)$ & 0 \\
\hline Total & Universidade Federal de Pernambuco & $1(5)$ \\
\hline
\end{tabular}

Fonte: Dados da pesquisa (2020).

Foram identificadas 20 produções científicas, entre os anos de 2010 e 2020, entre dissertações e teses sobre a dor crônica em hipertensos, destas, 16 são dissertações de mestrado e 04 teses de doutorado. Quanto ao número de achados, o ano de 2013, foi o ano de maior produção com 04 estudos, seguidos por 2014 e 2016 com 03 produções cada, 2011, 2015, 2017 e 2018 com 02 estudos respectivamente, 2010 e 2012 com apenas um. As regiões Sul, Sudeste e Centro-oeste apresentaram um equilíbrio no número de trabalhos, cada um com 06, seguido pela região Nordeste com apenas dois trabalhos, conforme explicitado na Tabela 01. Foram reconhecidos 19 programas de pós-graduações e desse total, a Universidade de São Paulo (USP), produziu maior número de pesquisas, divididos em: Tecnologias e atenção à saúde - Mestrado profissional; Mestrado em enfermagem na saúde do idoso; Mestrado em enfermagem fundamental e Mestrado em saúde coletiva, como mostrado na Tabela 1.

Quanto à formação profissional dos autores dos estudos, a enfermagem destacou-se como a profissão com maior número de produção ou defesas, com 06 estudos, medicina com 05, saúde coletiva com 04 trabalhos e os outros distribuídos entre: farmácia, educação física, gestão organizacional e ciências e tecnologias em saúde com um estudo em cada área. Sobre o desenho metodológico adotado nas pesquisas, destacou-se a abordagem transversal, com base populacional, descritiva e quantitativa em 15 produções. Em menor escala apareceram as pesquisas qualitativas, descritivas, observacionais e epidemiológicas. E ao final da leitura, optou-se pelo uso das palavras-chaves observadas nas teses e dissertações, as quais foram agrupadas, analisadas e destacadas para a construção de um mapa conceitual. O mapa foi identificado como "Dor Crônica e Hipertensão", para o qual foi dividido em dois eixos: predominância e sintomatologia, sendo a predominância, subdividida para o "Idoso", apresentado na Figura 2.

Portanto, apresentando-se de fácil entendimento para o leitor e considerado uma ferramenta de demonstração e organização de conhecimento relacionado entre conceitos que facilitam uma estratégica reflexão. Podem ser denominadas como unidades semânticas ou de sentido (Bittencourt et al., 2012). 
Figura 2. Mapa conceitual elaborado a partir dos descritores das teses e dissertações selecionadas para estudo, (2020).

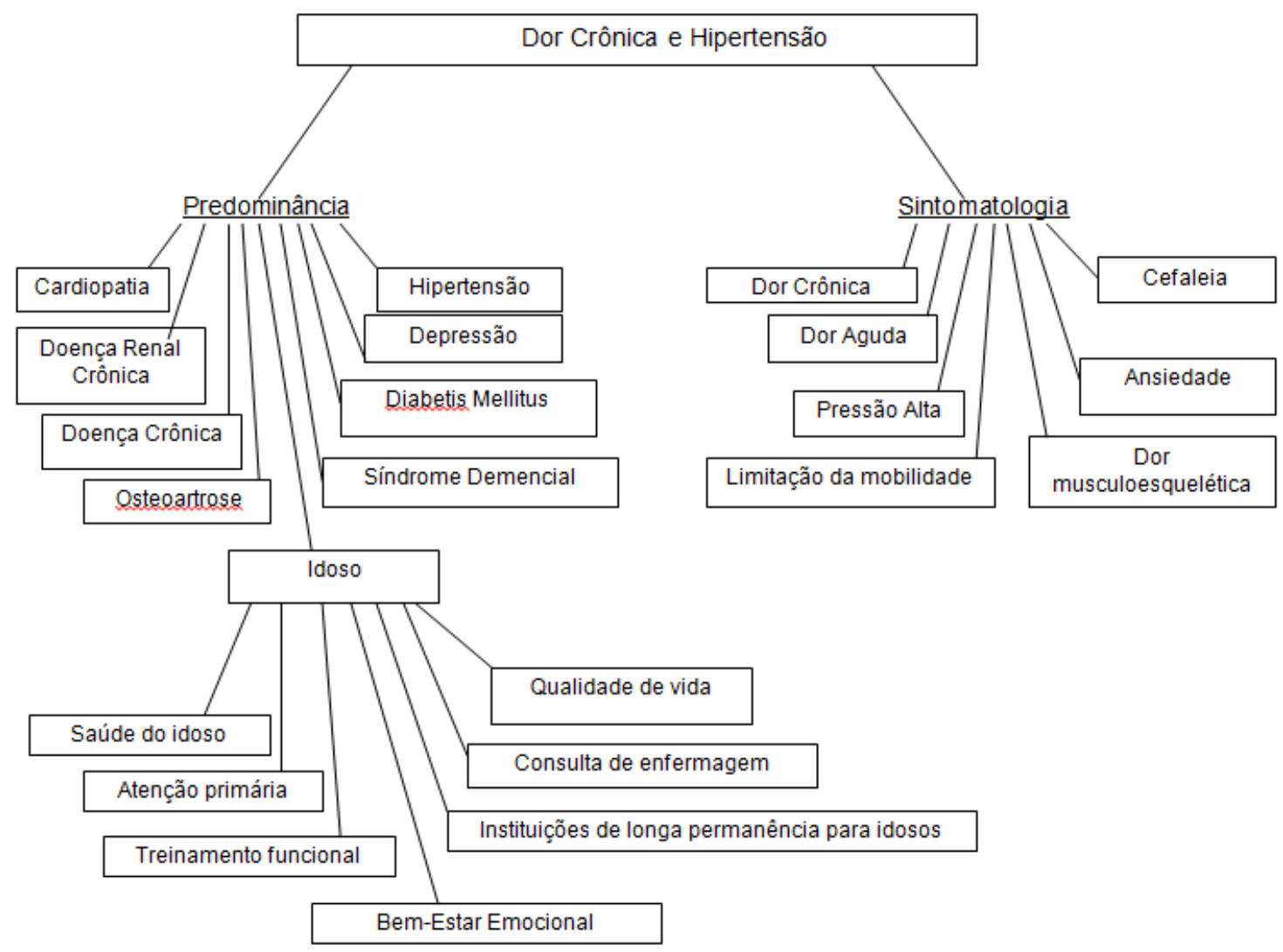

Fonte: Dados de Pesquisa (2020).

\section{Discussão}

Os achados obtidos mostram um equilíbrio no número de dissertações e teses a partir do ano de 2013, e é possível que o interesse sobre o assunto possa ser explicado pelo aumento da expectativa de vida brasileira e acerca da doença crônica. Quanto aos cenários, a predominância foi para os serviços de saúde de atenção básica, com a participação de 191 a 3035 sujeitos, em sua maioria do sexo feminino, com idades entre 18 e 90 anos. E as doenças de maior prevalência nos estudos foram: hipertensão, doença arterial coronariana, infarto, diabetes, doença renal crônica, depressão, dislipidemia, doenças osteomusculares, câncer e neuropatias diabéticas.

No que concerne ao desenho metodológico, à abordagem quantitativa, esteve presente em grande parte dos estudos. É um tipo de pesquisa capaz de mostrar a profunda natureza das realidades, seus sistemas de relações, sua estrutura dinâmica, além de fazer inferências causais 
que demonstram o motivo das coisas acontecerem ou não de uma forma determinada (Pita \& Pértegas, 2012).

Os mecanismos de relação entre a dor e a pressão arterial ainda não estão bem estabelecidos na literatura (Sacoò et al., 2013). A presença de patologias crônicas associadas ao quadro álgico pode ser um fator que prejudique o tratamento. Tal cenário pode dificultar a gestão do cuidado a esse paciente, pois o profissional pode relativizar um dos agravos, o que interfere diretamente na opção da terapêutica instituída, além do mais a fisiologia da pessoa na terceira idade passa por transformações e a medicação administrada, pode afetar no controle pressórico (Kaye et al., 2010).

A dor geralmente está associada a disfunções musculoesqueléticas e a lesão tecidual, interferindo de forma negativa na saúde do idoso (Dellaroza et al., 2012). Normalmente está interligada a processos patológicos crônicos que se prolongam por meses ou anos, as dores crônicas têm se tornado a principal queixa e causa de limitações funcionais nesses tipos de pessoas (Dellaroza et al., 2013), afetando de maneira importante a sua independência na realização das tarefas do cotidiano.

Foi identificado que há enfermeiros na produção de pesquisas sobre a temática, o que pode talvez ser explicado, devido o trabalho com o cuidado e a busca pela melhor maneira de lidar e tratar do próximo de forma individualizada e humanizada.

Segundo a Política Nacional de Saúde da Pessoa Idosa, o processo de envelhecimento deve ser ativo, pois o idoso deve estar ciente sobre os tipos de doenças em que está predisposto e sobre como manter-se saudável através de bons hábitos alimentares, exercícios físicos e um bem-estar emocional. Para que isso aconteça, é imprescindível que o acompanhamento e a promoção da saúde sejam de abordagem multidisciplinar e garantido pela atenção primária (Ministério da Saúde - Política Nacional de Saúde da Pessoa Idosa [PNSPI], 2006).

Estudos ressaltam e é observado na prática profissional que a equipe de enfermagem está diretamente ligada em todas as fases do tratamento, desde o diagnóstico inicial da doença, até a finitude (World Health Organization [WHO], 2018). Por esta razão deve-se valorizar a fala e as necessidades, ou seja, dar importância a dor que a pessoa está sentindo, ajudar para que seja cessada e por vezes apenas dar apoio no momento de angústia. Outro aspecto que merece destaque é a elaboração detalhada de um planejamento terapêutico individualizado, com a inserção da família como integrantes essenciais no ato do cuidado (Johnston et al., 2010). 
Research, Society and Development, v. 9, n. 12, e37191211033, 2020

(CC BY 4.0) | ISSN 2525-3409 | DOI: http://dx.doi.org/10.33448/rsd-v9i12.11033

Vale ressaltar que as políticas públicas, como também a atenção ao cuidado, o manejo e o tratamento aos idosos tanto por parte por parte da família quanto dos serviços de saúde devem melhorar, pois o crescimento desta população é uma realidade e as doenças crônicas geralmente são preveníveis podendo reduzir transtornos para todos, economia aos cofres públicos e principalmente a dignidade no processo de envelhecimento do ser humano.

\section{Considerações Finais}

Durante a pesquisa, foi observado um pequeno número de estudos provenientes de dissertações e teses sobre a relação da dor crônica em hipertensos, defendidas em Programas de Pós-Graduação no país. E dada a relevância do tema, faz-se necessário novas investigações e a disseminação de evidências no cenário da prática profissional.

\section{Referências}

Albuquerque, F. Dor crônica afeta pelo menos $37 \%$ dos brasileiros. Agência Brasil. Recuperado de: https://agenciabrasil.ebc.com.br/saude/noticia/2018-09/dor-cronica-afetapelo-menos-37-dos-brasileiros.

Alves, A. M., Costa, S. F., Fernandes, M. A., Batista, P. S., Lopes, M. E., \& Zaccara, A. A. (2019). Communication in palliativecare: a bibliometricstudy. Journal Res Fundam Care Online; 11(2). Recuperado de: http://dx.doi.org/10.9789/2175-5361.2019.v11i2.524-532.

Bittencourt, G. K., da Nóbrega, M. M., Medeiros, A. C., \& Furtado, L. G. (2013). Mapas conceituais no ensino de pós-graduação em enfermagem: relato de experiência. Revista Gaúcha Enfermagem, 34(2). Recuperado de: https://www.scielo.br/pdf/rg enf/v34n2/v34n2a22.pdf.

Brandão, A. A., \& Nogueira, A. R. (2018). Manual de Hipertensão Arterial. Rio de Janeiro: Sociedade Brasileira de Cardiologia do estado do Rio de Janeiro - SOCERJ. Recuperado de https://socerj.org.br/antigo/wp-content/uploads/2018/04/Manual_Hipertensa\%CC\%83o_A terial_Completo_Final.pdf. 
Dellaroza, M. S., \& Pimenta, C. A. (2012). Impacto da dor crônica nas atividades de vida diária de idosos da comunidade. Ciência Cuidado Saúde. Recuperado de: http://www.observatorionacionaldoidoso.fiocruz.br/biblioteca/_artigos/144.pdf.

Dellaroza, M. S., Pimenta, C. A., Duarte, Y. A., \& Lebrão, M. L. (2013). Chronic pain among elderly residents in São Paulo, Brazil: prevalence, characteristics, and association with functional capacity and mobility (SABE Study). Caderno Saúde Pública. 29(2): https://doi.org/10.1590/S0102-311X2013000200019.

Dias, K. C. C. O., Batista, P. S. S., Fernandes, M. A., Zaccara, A. A. L., Oliveira, T. C., \& Vasconcelos M. F de. (2019) Dissertações e teses sobre cuidados paliativos em oncologia pediátrica: estudo bibliométrico. Acta paulista de enfermagem. https://acta-ape.org/wpcontent/uploads/articles_xml/1982-0194-ape-33-eAPE20190264/1982-0194-ape-33eAPE20190264.pdf.

Freitas, R. S., Fernandes, M. H., Coqueiro, R. S., Reis, W. J., \& Rocha, S., Brito A. (2012) Capacidade funcional e fatores associados em idosos: estudo populacional. Acta Paulista de Enfermagem, 25(6). Recuperado de: https://www.scielo.br/pdf/ape/v25n6/v25n6a17.pdf.

Galvão, T. F, Pansani, T. S, \& Harrad, D. (2015). Principais itens para relatar revisões sistemáticas e meta-análises: a recomendação PRISMA. Epidemiol Serv Saude. 24(2). http://dx.doi.org/10.5123/S1679-49742015000200017.

Hlubocky, F. J., Muffly, L. S., Gomez, J. X., Breitenbach, K., Cella, D., \& McNeer, J. L. (2016). Death anxiety, psychological distress, and quality of life (QOL) in adolescent and young adult (AYA) cancer patients with hematologic malignancies in early survivorship. Journal Clin Oncol. 34(14). Recuperado de: https://ascopubs.org/doi/abs/10.1200/ JCO.2016.34.15_suppl.10073.

Johnston, D. L., Nagel, K., Friedman, D. L., Meza, J. L., Hurwitz, C. A., \& Friebert, S. (2010). Availability and use of palliative care and end-of-life services for pediatric oncology patients. Journal Clin Oncol. 26(28). Recuperado de: https://ascopubs.org/doi/10.1200/JC O.2008.16.1562?url_ver=Z39.882003\&rfr_id=ori\%3Arid\%3Acrossref.org\&rfr_dat=cr_pub+ +0pubmed\&. 
Kaye, A. D., Baluch, A., \& Scott, J. T. (2010). Pain management in the elderly population: a review. The Ochsner Journal. Recuperado de: https://www.ncbi.nlm.nih.gov/pmc/article s/PMC3096211/pdf/i1524-5012-10-3-179.pdf.

Marcelo, J. F. \& Hayashi, M. C. P. I. (2013). Estudo bibliométrico sobre a produção científica no campo da sociologia da ciência. Informação \& Informação. Recuperado de: http://dx.doi.org/10.5433/1981-8920.2013v18n3p138.

Ministério da Saúde (2006). Política Nacional de Saúde da Pessoa Idosa. Brasília: Ministério da Saúde. Recuperado de: https://bvsms.saude.gov.br/bvs/saudelegis/gm /2006/prt2528_19_10_2006.html.

Pita, F. S., \& Pértegas, D. S. (2012). Investigación cuantitativa y cualitativa. Caderno Atenção Primaria. (76)8. Recuperado de: http://www.fisterra.com/gestor/upload/gui as/cuanti_cuali2.pdf.

Sacoò, M., Meschi, M., Regolisti, G., Detrenis, S., Bianchi, L., \& Bertorelli, M.(2013). The relationship between blood pressure and pain. Journal Clinic Hypertension. Recuperado de: https://pubmed.ncbi.nlm.nih.gov/23889724/.

Witte, W., \& Stein, C. (2010). History, Definitions and Contemporary Viewpoins. In: Kopf A, Patel NB, editors. Guide to pain Management in Low-Resource Settings. Seattle; IASP; 2010. (3)8. Recuperado de: https://s3.amazonaws.com/rdcms-iasp/files/production/public/Conte nt/ContentFolders/Publications2/FreeBooks/Guide_to_Pain_Management_in_Low-Resource _S ettings.pdf.

World Health Organization (2018). Recuperado de: https://www.who.int/data/gho/whs-2020visual-summary.

\section{Porcentagem de contribuição de cada autor no manuscrito}

Valeriana Cantanhede Rodrigues - 65\%

Dayse Mary da Silva Correia- 35\% 